\title{
Background-Free Microwave Signal Generation Based on Unbalanced Temporal Pulse Shaping
}

\author{
Wen Ting Wang, Ming Li, Shu Qian Sun, Chao Wang, Ye Deng, and Ning Hua Zhu
}

\begin{abstract}
We propose a new 1 method to generate background-free high-frequency pulsed microwave signal based on an unbalanced temporal optical pulse shaping (TPS) system and balanced photodetection. The proposed system consists of a polarization modulator and an unbalanced TPS system realized by two conjugate dispersion elements. The carrier frequency of the pulsed microwave signal could be tuned by changing the residual dispersion of the TPS. The proposed method is theoretically analyzed and experimentally demonstrated. The experimental results show that the carrier frequency of the generated microwave pulse could be tuned over a broad frequency range. Moreover, the generated microwave pulse signal is background-free by suppressing the baseband frequency components using balanced photodetection.
\end{abstract}

Index Terms-Pulsed microwave signal, unbalanced temporal pulse shaping, balanced photo-detection.

\section{INTRODUCTION}

$\mathrm{P}$ HOTONIC generation of pulsed microwave signals has attracted considerable attention for its widespread applications in radar systems, microwave tomography, broadband wireless access networks, and warfare systems [1-3]. Compared with its electrical counterparts, photonic generation of microwave signals has some advantages such as wide bandwidth, low loss transmission, and low power consumption, and immunity to electromagnetic interference (EMI) [4]. Up to now, various configurations have been reported to generate pulsed microwave signals. High frequency microwave pulse signals are generated by simply multiplying the low frequency microwave signal and then truncating the microwave signal into pulsed microwave signals [5]. High frequency microwave signals are also generated based on optical spectral-shaping incorporating with frequency-to-time mapping [6], which is a powerful and

Manuscript received November 10, 2015; revised January 3, 2016, accepted January 4, 2016. This work was supported by the National Natural Science Foundation of China under 61377069, 61335005, 61321063, and 61090391.

W. T. Wang is with the State Key Laboratory on Integrated Optoelectronics, Institute of Semiconductors, Chinese Academy of Sciences, Beijing 100083, China, and also with the Center for Free-Electron Laser Science, Deutsches Elektronen-Synchrotron, Hamburg 22607, Germany (e-mail: wtwang13@semi.ac.cn).

M. Li, S. Q. Sun, Y. Deng, and N. H. Zhu are with the State Key Laboratory on Integrated Optoelectronics, Institute of Semiconductors, Chinese Academy of Sciences, Beijing 100083, China (e-mail: ml@semi.ac.cn; sqsun@semi.ac.cn; ydeng@semi.ac.cn; nhzhu@semi.ac.cn).

C. Wang is with the School of Engineering and Digital Arts, University of Kent, Canterbury CT27NT, U.K. (e-mail: c.wang@kent.ac.uk). promising method. The key component in these systems is an optical spectral shaper, which is usually realized using a fiber comb filter or a spatial light modulator (SLM) [7]. The advantage of using a SLM is that its transmission response is flexibly reconfigurable in real time. Therefore, an arbitrary microwave waveform could be easily generated using SLM-based system. However, the key limitation of the SLM based microwave pulse generation system is high loss and poor long-term stability as it involves space-to-fiber and fiber-to-space coupling. As an alternative candidate for spectral shapers, a fiber comb filter is smaller, with higher stability and lower power consummation [8]. However, the spectral response keeps unchanged once it is fabricated. C. Wang proposed and experimentally demonstrated a powerful method to generate a frequency-multiplied microwave signal using a unbalanced temporal pulse shaping system, which consists of two dispersive elements with opposite dispersion but non-identical in magnitude and an electro-optic modulator located between the two dispersive elements [9]. One of the limitations in this system is that the generated microwave pulse has baseband frequency components in the electrical spectrum which would interfere with other narrow-band serves. J.D. Mckinney proposed a powerful method to eliminate the baseband components using polarization pulse shaping. However, the proposed system is bulky [10].

In this letter, we propose a new method to generate background-free pulsed microwave signals based on unbalanced TPS and balanced photo-detection. A length of dispersion compensating fiber (DCF) and single mode fiber (SMF) are used as two dispersive elements in this system. A joint use of a PolM, a Polarization controller (PC) and a polarization beam splitter (PBS) is equivalent to two intensity modulators with differential response. The frequency of the pulsed microwave signal could be tuned by changing the residual dispersion of the TPS. The experimental results show that the frequency of the generated microwave pulse could be tuned. Moreover, the generated microwave pulse signal is background-free by suppressing the baseband frequency components using balanced photo-detection.

\section{PRINCIPAL}

The schematic diagram of the proposed background-free pulsed microwave signal generation system based on unbalanced temporal pulse shaping and balanced photo-detection is shown in Fig. 1. A transform-limited ultra-short optical pulse 
emitted from a mode-locked laser (MLL) is firstly sent to a dispersion compensating fiber (DCF), which is used to stretch the optical pulse in the time domain. The stretched optical pulse is then injected into the PolM, where its polarization state is modulated by a low-frequency microwave drive signal generated by an arbitrary waveform generator (AWG). A polarizer is integrated at the input of the PolM with an angle of 45 deg with respect to the principle axis of the PolM. A PC1 attached after the DCF is used to adjust the polarization state of the optical pulse to reduce the polarization dependence loss. The polarization modulated optical signal is then compressed by the conjugated dispersion components. In our scheme, a length of single mode fiber (SMF) is used as a dispersion element to compress the modulated signal. The joint use of the PolM, the PC2 and the PBS is equivalent to an intensity modulator. A continuous microwave signal emitted from the AWG is launched into the radio frequency port of the PolM. The center frequency of the generated microwave pulse signal is mainly determined by the residual dispersion value of the UDS for the given microwave drive signal. The two output signals at the output of the PBS are then fed into the balanced photo-detector (BPD) to achieve optical-to-electrical conversion. Electrical

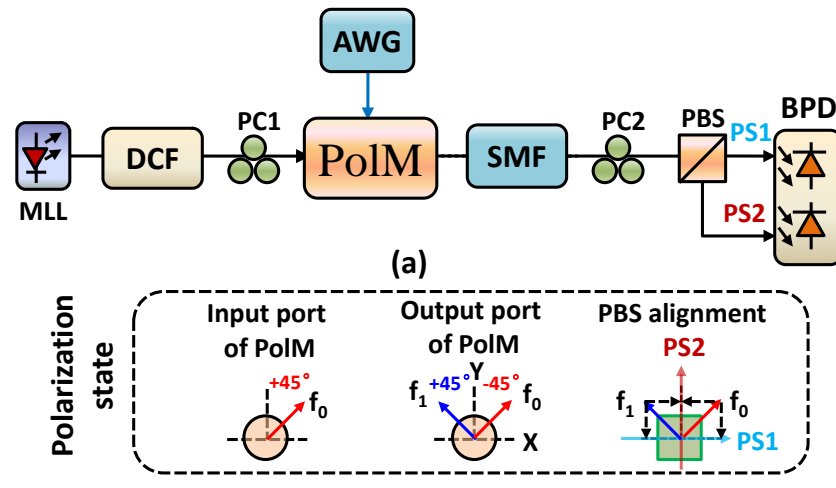

(b)

Fig. 1. The schematic diagram of the proposed system for background-free pulsed microwave signal. MLL: mode-locked laser; DCF: dispersion compensating fiber; PC1, PC2: polarization controller; AWG: arbitrary waveform generator; PolM: polarization modulator; SMF: single mode fiber; PBS: polarization beam splitter; BPD: balanced photo-detector.

spectrum of the generated microwave pulse signal is recorded by an electrical spectrum analyzer (ESA) and an oscilloscope (OSC) is used to capture the temporal waveform.

Mathematically, the transform-limited Gaussian pulse in the time domain could be expressed as $\mathrm{g}(\mathrm{t})=\exp \left(-\mathrm{t}^{2} / \tau^{2}\right)$, where $\tau$ is the half pulsewidth at the 1/e maximum. The ultra-short optical pulse is firstly sent into the first dispersive medium assumed to provide only first-order standard dispersion. The normalized transfer function of the first and second dispersive elements could be represented as $H_{1}(\omega)=\exp \left(j \ddot{\Phi}_{1} \omega^{2} / 2\right)$ and $H_{2}(\omega)=$ $\exp \left(\mathrm{j} \ddot{\Phi}_{2} \omega^{2} / 2\right)$ when the higher order dispersion is ignored. In our scheme, $\ddot{\Phi}_{1}$ and $\ddot{\Phi}_{2}$ are group velocity dispersion of the DCF and the SMF, respectively. A sinusoidal microwave signal is then applied to the PolM. A joint use of the PolM with a PC and a PBS is equivalent to an intensity modulator. Under small-signal modulation condition, the modulation function of the PolM could be represented as $\operatorname{EPolM}_{\mathrm{P}}(\mathrm{t})=\mathrm{J}_{0}(\mathrm{~m})+2 \mathrm{~J}_{1}(\mathrm{~m}) \times$ $\cos \left(\mathrm{i} \omega_{\mathrm{RF}} \mathrm{t}\right)$ where $J_{n}(\cdot)$ is the first-order Bessel function of the first kind, $\omega_{\mathrm{RF}}$ is the angular frequency of the driven microwave signal and $\mathrm{m}$ is the phase modulation index. The optical signal at the output of PolM could be represented as

$$
s(t) \approx g(t) \times\left(J_{0}(m)+2 J_{1}(m) \cos \left(\omega_{R F} t\right)\right)
$$

A pair of dispersive components with conjugated dispersion value is used to realize temporal pulse shaping. The second dispersive element assumed to provide anomalous dispersion followed after the PolM is used to compress the polarization modulated optical pulse. The modulated and compressed optical pulse at the output of the second dispersive element could be rewritten as follows:

$$
\begin{aligned}
f(t)= & \exp \left(\frac{j}{2 \Delta \ddot{\Phi}} t^{2}\right) J_{0}(m)(\Im[g(t)])_{\omega=t / \Delta \ddot{\Phi}}+ \\
& \exp \left(\frac{j}{2 \Delta \ddot{\Phi}} t^{2}\right) J_{1}(m)(\Im[g(t)])_{\omega=t / \Delta \Phi} \cos \left(\frac{\ddot{\Phi}_{1}}{\Delta \ddot{\Phi}} \omega_{R F} t\right)
\end{aligned}
$$

where $\Delta \ddot{\Phi}=\ddot{\Phi}_{1}-\ddot{\Phi}_{2}$ is defined the residual dispersion of unbalanced TPS, and the $\mathfrak{I}$ is the Fourier transform. If the optical signal at the output of the second dispersive element is applied into a single PD, we could have the photocurrent as follow:

$$
\begin{aligned}
& I(t) \propto J_{0}^{2}(m)(\Im[s(t)])_{\omega=t / \Delta \ddot{\Phi}}^{2}+J_{1}^{2}(m)(\Im[s(t)])_{\omega=t / \Delta \ddot{\Phi}}^{2} \\
& +2 J_{0}(m) J_{1}(m)(\mathfrak{\Im}[s(t)])_{\omega=t / \Delta \ddot{\Phi}}^{2} \cos \left(\frac{\ddot{\Phi}_{1}}{\Delta \ddot{\Phi}} \omega_{R F} t\right) \\
& +J_{1}^{2}(m)(\Im[s(t)])_{\omega=t / \Delta \ddot{\Phi}}^{2} \cos \left(\frac{2 \ddot{\Phi}_{1}}{\Delta \ddot{\Phi}} \omega_{R F} t\right)
\end{aligned}
$$

From Eq. (3), we could draw a conclusion that the frequency of the generated microwave signal could be continuously tuned by controlling the residual dispersion and the frequency-doubled microwave pulse could be generated.

It is worth noting that the previously proposed method based on temporal pulse shaping generates microwave pulses with a baseband frequency components originated from the broadband optical spectrum profile. In order to generate a background-free pulsed microwave signal, the optical signals at the output of the PBS is divided into two parts and fed into the BPD. The PolM is a special phase modulator that could support both TE and TM modes with opposite phase modulation indices. The linearly polarized optical signal is oriented at an angle of 45 deg to one principle axis of the PolM denoting with a symbol of $\mathrm{X}$. The spectra of the modulated optical signals include the optical carrier and the correspondingly modulated sidebands which are orthogonal with each other orienting with the axis of $\mathrm{X}$ of 45and 135- deg, respectively.

Assuming only a single optical carrier is injected into the PolM. The PolM is a special phase modulator to support on both transverse electric (TE) and transverse magnitude (TM) modes with opposite phase modulation indices. The output optical filed of the PolM could be expressed as: 


$$
E_{\text {PolM }}=\left[\begin{array}{l}
E_{x} \\
E_{y}
\end{array}\right]=\frac{E_{\text {in }}}{\sqrt{2}}\left[\begin{array}{l}
\exp \left(j m \cos \left(2 \pi f_{R F} t\right)\right) \\
\exp \left(-j m \cos \left(2 \pi f_{R F} t\right)-j \theta\right)
\end{array}\right]
$$

where $E_{\text {in }}=E_{0} \exp \left(i 2 \pi f_{0} t\right)$ is the optical field at the input of the PolM. $\theta$ is the phase difference between $E_{x}$ and $E_{y}$ which could be controlled by the dc bias of the PolM. $m=\pi \mathrm{V}_{\mathrm{RF}} / \mathrm{V}_{\pi}$ is the phase modulation index of the PolM and the $\mathrm{V}_{\pi}$ is the half-wave voltage of the PolM. $\omega_{\mathrm{RF}}$ is the angular frequency of the driven microwave signal. Appling Jacobi-Anger expansion to Eq. (4) and considering small signal modulation condition, we have

$$
E_{\text {PolM }}=\left[\begin{array}{l}
E_{x} \\
E_{y}
\end{array}\right]=\frac{E_{\text {in }}}{\sqrt{2}}\left[\begin{array}{l}
J_{0}(m)+J_{1}(m) \exp j\left(2 \pi f_{R F} t+\pi / 2\right)+ \\
J_{1}(m) \exp j\left(-2 \pi f_{R F} t+\pi / 2\right) \\
J_{0}(m)-J_{1}(m) \exp j\left(2 \pi f_{R F} t+\pi / 2\right)- \\
J_{1}(m) \exp j\left(-2 \pi f_{R F} t+\pi / 2\right)
\end{array}\right]
$$

where $\mathrm{J}_{\mathrm{n}}$ is the Bessel function of the first kind of order $\mathrm{n}$. We assume $\theta=0$. It is worth noting the optical carrier and sidebands are polarized at 45 degree and -45 degree to X-axis of the PolM. These modulated components then pass through the PC2 and feed into the PBS. The optical filed of the modulated signal could be rewritten as follows:

$$
\left[\begin{array}{c}
E_{x+45^{\circ}} \\
E_{x+135^{\circ}}
\end{array}\right]=\frac{E_{i n}}{\sqrt{2}}\left[\begin{array}{c}
J_{0}(m) \\
J_{1}(m) \exp j\left(2 \pi f_{R F} t+\pi / 2\right)+ \\
J_{1}(m) \exp j\left(-2 \pi f_{R F} t+\pi / 2\right)
\end{array}\right]
$$

By tuning the PC2, the X-axis of the PolM is aligned the polarized state 1 (PS1).and the Y-axis of that is aligned the polarized state 2 (PS2). Therefore, the optical filed at the PS1 and PS2 could be written as follows:

$$
\left[\begin{array}{c}
E_{P S 1} \\
E_{P S 2}
\end{array}\right]=\frac{\sqrt{2}}{2}\left[\begin{array}{l}
E_{x+45^{\circ}}-E_{x+135^{\circ}} \\
E_{x+45^{\circ}}+E_{x+135^{\circ}}
\end{array}\right]
$$

After the PBS, the optical signal is detected in the PD, resulting in photocurrents as follows

$$
\left[\begin{array}{l}
I_{P S 1} \\
I_{P S 2}
\end{array}\right] \propto\left[\begin{array}{l}
J_{0}^{2}(m)+2 J_{1}^{2}(m)-4 J_{0}(m) J_{1}(m) \cos \left(\omega_{R F} t+\pi\right) \\
J_{0}^{2}(m)+2 J_{1}^{2}(m)-4 J_{0}(m) J_{1}(m) \cos \left(\omega_{R F} t\right)
\end{array}\right]
$$

When the two output signals from the PBS is injected into a BPD for balanced photo-detection, the photocurrent could be represented as follow

$$
I_{B P D} \propto 8 J_{0}(m) J_{1}(m) \cos \left(\omega_{R F} t\right)
$$

Therefore, when the continuous wave optical carrier is replaced by a stretched optical pulse, the photocurrent at the output of the BPD is

$$
\begin{aligned}
I_{B P D}(t)= & 4 J_{0}(m) J_{1}(m)(\Im[s(t)])_{\omega=t / \Delta \ddot{\Phi}}^{2} \cos \left(\frac{\ddot{\Phi}_{1}}{\Delta \ddot{\Phi}} \omega_{R F} t\right) \\
& +2 J_{1}^{2}(m)(\Im[s(t)])_{\omega=t / \Delta \ddot{\Phi}}^{2} \cos \left(\frac{2 \ddot{\Phi}_{1}}{\Delta \ddot{\Phi}} \omega_{R F} t\right)
\end{aligned}
$$

It can be seen from Eq. (10) that the signal to noise ratio (SNR) of the generated microwave signals is improved by 4 times. Moreover, the baseband frequency components of the generated microwave pulse in the Eq. (10) are eliminated by the balanced photo-detection. The frequency of the generated microwave signal is equal to $\ddot{\Phi}_{1} / \Delta \ddot{\Phi} \times \omega_{R F}$ such that the frequency of the carrier frequency of the pulsed microwave signal
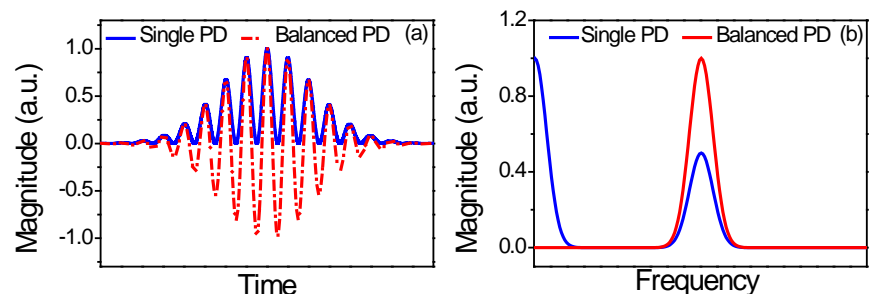

Fig. 2. Simulation results for the microwave pulse generation, (a) the simulated electrical waveform, (b) the simulated electrical spectrum.

could be tuned by simply changing the residual dispersion. The Fig 2 shows the simulated results. Fig 2 (a) shows the waveform of the generated microwave pulse. The waveform in the rod dash line is generated bases on balanced photo-detection and that in the blue solid line is generated based on single-end detection. The corresponding Fourier transform spectra are shown in Fig. 2 (b). We could obtain the high frequency microwave pulse for both the single-end detection and the balanced detection. However, it is obviously observed that strong low-frequency components appear in single-end detection case and the amplitude of the generated microwave pulse is half of that of the microwave pulse achieved by the balanced detection. Therefore, the balanced photo-detection has higher power efficiency and no strong low frequency components. The main significance of the proposed method is that the frequency of the pulsed microwave signal could be continuously tuned by changing the dispersion value of the dispersive element. The generated microwave signal is background free and the power of that is enhanced. Moreover, when the linearly chirped dispersive element is explored in the UDS, the linearly chirped microwave pulse with long duration time could be generated [11]. The chirped pulsed microwave is very important in the radar system [9].

\section{EXPERIMENT}

We carried out experiments based on the setup shown in Fig. 1 to verify the proposed scheme. An MLL (Pritel) is used to emit an ultra-short optical pulse train with an average output power of $29.7 \mathrm{~mW}$ and a pulse-width of $120 \mathrm{fs}$ and a pulse repetition rate of $50.8 \mathrm{MHz}$. The PolM has a 3-dB bandwidth of $40 \mathrm{GHz}$ and a half-wave voltage of $3.5 \mathrm{~V}$. A sinusoidal microwave signal emitting from an AWG with an output power of $-10 \mathrm{dBm}$ is injected into the PolM such that small signal modulation condition is fulfilled. The DCF and SMF are used as the first
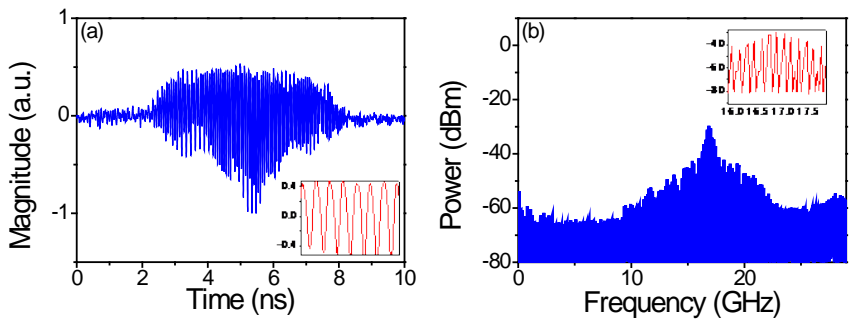

Fig. 3. Measured (a) the generated electrical waveform, (b) the generated electrical spectrum. Insets show the zoom-in views.

and the second dispersive elements, respectively. They form a pair of dispersive elements as required in the unbalanced tem- 
poral pulse shaper to process the optical pulse. The group velocity delay (GVD) of the DCF is not matched to that of the SMF. The BPD has a bandwidth of $40 \mathrm{GHz}$ and a conversion gain of $110 \mathrm{~V} / \mathrm{W}$. The generated microwave pulse signal is recorded by a high-speed oscilloscope. Its electrical spectrum is measured by an electrical spectrum analyzer (ESA). Fig. 3 (a) shows the generated waveform obtained by the balanced photo-detection, which is actually the amplitude summation of the two generated waveforms in the two PD of the BPD. It is obviously observed by Fig 3 (a) that the amplitude of the generated microwave pulse is nearly twice of that with a single PD. The insets of the Fig 3 (a) and 3 (b) are the zoom in the waveform and electrical spectrum, respectively. As can be seen from the inset of the Fig 3 (a), the waveform has both positive and negative parts and not a pedestal. Theoretically, the waveform of the generated microwave pulse should have both positive and negative parts with identical amplitude. It is clearly seen from the Fig. 3 (a) that the waveform has asymmetrical positive and negative parts which is mainly attributed to the unequal input optical power for the BPD induced by the polarization drift or the different responsivity of the two PDs in the BPD. The duration of generated microwave pulse is about $2.2 \mathrm{~ns}$. The temporal oscillation period of the generated microwave pulse is about 59 ps, which is corresponding to a carrier frequency of 16.949 GHz. Fig.3 (b) shows the measured electrical spectrum of the generated microwave pulse. The center frequency is 16.9 GHz. As can be seen from Fig. 3 (b), the baseband frequency

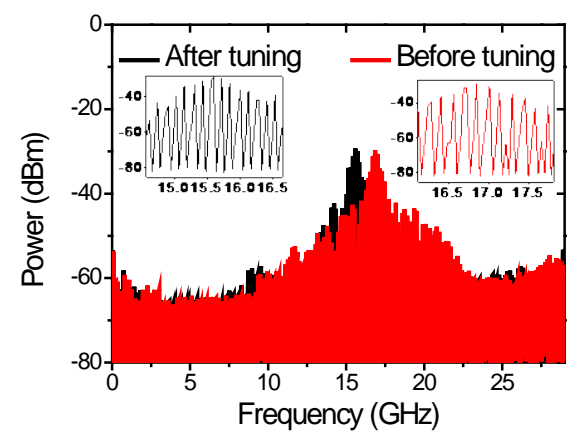

Fig. 4. Measured electrical spectra before tuning and after tuning. Insets show the zoom-in views.

components are nearly eliminated by the balanced photo-detection. Therefore, the background-free pulsed microwave signal is successfully obtained by the proposed method.

In order to demonstrate the frequency tunability of the generated microwave signals, the residual dispersion of the system is tuned by changing the length of the SMF. Fig. 4 shows the electrical spectra of the generated microwave signal. As can be seen from Fig. 4, the center frequency of the generated microwave signal has been tuned to $15.5 \mathrm{GHz}$ in black line. The insets in the Fig.4 are the zoom in the obtained spectra. It is successfully demonstrated that by properly changing the residual dispersion value of the UDS, the frequency of the generated microwave pulse signal could be flexibly tuned.

\section{CONCLUSION}

We have proposed and experimentally demonstrated a new method to generate background-free pulsed microwave signals with tunable center frequency based on unbalanced temporal pulse shaping and balanced photo-detection. The ultra-short optical pulse is temporally processed by the temporal pulse shaper using a UDS. Since the two dispersive components are not matched in GVD, the entire system is equivalent to a balanced temporal pulse shaper followed by a residual dispersive processor for a second real-time Fourier transform (Fresnel diffraction). The center frequency of the generated pulsed microwave signal is tuned by changing the residual dispersive value of the unbalanced temporal shaping system. The experimental results show that the generated microwave pulse has a temporal oscillation period of 59 ps and the corresponding center frequency of that is $16.9 \mathrm{GHz}$. It is worth noting that the electrical spectrum of the generated microwave pulse signal has not baseband frequency components. Moreover, the center frequency of the generated microwave signal could be tuned.

\section{REFERENCES}

[1] .J. Azana, N. K. Berger, B. Levit, and B. Fischer, "Reconfigurable generation of high-repetition-rate optical pulse sequences based on time-domain phase-only filtering," Opt. Lett., vol. 30, no.23, pp. 3228-3231, Dec. 2005.

[2] M. A. Muriel, J. Azana, and A. Carballar, "Real-time Fourier transformer based on fiber gratings,” Opt. Lett., vol. 24, no. 1, pp. 1-3, Jan. 1999.

[3] P. C. Chou, H. A. Haus, and J. F. Brennan III, "Reconfigurable time-domain spectral shaping of an optical pulse stretched by a fiber Bragg grating,” Opt. Lett., vol. 25, no.8, pp. 524-526, Apr. 2000.

[4] R. E. Saperstein, D. Panasenko, and Y. Fainman, "Demonstration of a microwave spectrum analyzer based on time-domain optical processing in fiber,” Opt. Lett., vol. 29, no.5, pp. 501-503, Mar. 2004.

[5] F. Z. Zhang, X. Z. Ge, S. L. Pan, and J. P. Yao, "Photonic generation of pulsed microwave signals with tunable frequency and phase based on spectral-shaping and frequency-to-time mapping," Opt. Lett., vol. 38, no.20, pp. 4256-4259, Oct. 2013.

[6] H. Chi, and J. P. Yao, "All-fiber chirped microwave pulses generation based on spectral shaping and wavelength-to-time conversion," IEEE Trans. Microw. Theory Tech., vol. 55, no. 9, pp. 1958-1963, Sep. 2009.

[7] N. K. Berger, B. Levit, B. Fischer, and J. Azana, "Picosecond flat-top pulse generation by low-bandwidth electro-optic sinusoidal phase modulation,” Opt. Lett., vol. 33, no.2, pp. 125-127, Jan. 2008.

[8] Y. Park, and J. Azana, "Optical signal processors based on a time-spectrum convolution,” Opt. Lett., vol. 35, no.6, pp. 796-798, Mar. 2010.

[9] C. Wang, M. Li, and J. P. Yao, “Continuously tunable photonic microwave frequency multiplication by use of an unbalanced temporal pulse shaping system," IEEE Photon. Technol. Lett., vol. 22, no. 17, pp. 1285-1288, Sep. 2010.

[10] W. T. Wang, W. Li, W. H. Sun, J. G. Liu, H. Q. Yuan, and N. H. Zhu, "Reconfigurable microwave photonic filter based on tunable dispersion-induced power fading in a dispersive element," Opt. Commun., vol. 333, pp. 209-212, Nov. 2015.

[11] M. Li, C. Wang, W. Z. Li, and J. P. Yao, "An unbalanced temporal pulse-shaping system for chirped microwave waveform generation," IEEE Trans. Microw. Theory Tech., vol. 58, no. 11, pp. 2968-2975, Nov. 2010. 